\title{
ORIGINAL ARTICLE \\ Assessment of in vivo spinal cord conduction velocity in rats in an experimental model of ischemic spinal cord injury
}

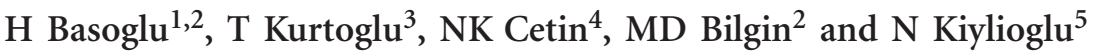

\begin{abstract}
Study design: Experimental laboratory investigation of spinal cord conductivity alterations in a rat model of ischemic spinal cord injury (SCl).

Objective: To observe the epidural spinal cord stimulation-induced electromyography responses, and to investigate the possible alterations of spinal cord conduction velocity (SCCV) and compound muscle action potentials (CMAPs) after ischemic SCl in rats.

Settings: Adnan Menderes University, Institute of Health Science, Aydin, Turkey.

Methods: SCl was induced by transient occlusion of the abdominal aorta in male Sprague-Dawley rats. Spinal cord histopathology was examined to determine neuronal damage and Tarlov scale was used to grade locomotor functions. Epidural electrical stimulation of spinal cord was performed by monopolar needle electrodes sequentially at L1-L2 and L5-L6 levels, and CMAPs were recorded from the left gastrocnemius muscle by surface electrodes. Amplitudes and durations of CMAPs were evaluated and SCCVs were calculated by analyzing the latency difference of CMAPs.

Results: Ischemia-induced $\mathrm{SCl}$ resulted in significant reduction of Tarlov scores and a significant decline in number of viable neurons. Similarly, a significant decrement was observed in SCCV following spinal cord ischemia.

Conclusion: This study demonstrated that measurement of SCCV via epidural electrical stimulation is possible and displays a significant decline after spinal cord ischemia in rats. We suggest that this method can be beneficial to quantify neuronal damage after experimental ischemic SCl.
\end{abstract}

Spinal Cord (2013) 51, 616-622; doi:10.1038/sc.2013.40; published online 21 May 2013

Keywords: spinal cord injury; electromyography; conduction velocity

\section{INTRODUCTION}

Spinal cord injury (SCI) is estimated to occur at an annual rate of 20-40 individuals per million in most countries and frequently results in irreversible loss of motor and sensorial functions. ${ }^{1}$ SCI occupies an important place in neurological research, and assessment of SCI by objective methods is crucial for constituting reliable and reproducible experimental designs. Such methods enable the researchers to determine the effect of various promising interventions and promote translation of preclinical experiments to human trials.

Rats are commonly used in experimental studies owing to the firm analogy of spinal cord anatomy between these animals and humans. ${ }^{2}$ Motor functions after SCI are generally evaluated by locomotor scales such as Basso Beattie and Bresnahan (BBB) and Tarlov scales. These qualitative assessment methods are performed by visual inspection and scoring of hind limb activity, and mainly determine fine locomotor skills. ${ }^{3}$ However, there are difficulties in objective determination of neurological status following SCI in rats, which drive the attempts for developing more quantitative methods. ${ }^{4,5}$ Various alternative methods have been developed for quantitative determination of motor deficits after SCI in rodents, which involve employment of video assistance such as single-frame motion analysis, ${ }^{5}$ automated quantitative gait analysis ${ }^{4}$ or robotic devices. ${ }^{6}$
Electromyography (EMG) is a technique for evaluating and recording the electrical activity produced by skeletal muscles. ${ }^{7}$ Electromyographic activity obtained from flexor and extensor muscles on walking has been used for quantitative determination of locomotor recovery after SCI in rats. ${ }^{8,9}$ Epidural electrical stimulation has been performed in rat models of SCI to facilitate posture and locomotion, ${ }^{10,11}$ to measure spinal cord-evoked potentials ${ }^{12}$ and to alleviate certain forms of neuropathic pain. ${ }^{13}$ Magnuson et al. ${ }^{14}$ used transcranial magnetic stimulation to generate motor-evoked potentials, recorded EMG responses by inserting subdermal needle electrodes into the gastrocnemius muscle, and observed prolonged latency in a contusion model of SCI. Kose and Basoglu ${ }^{15}$ reported that spinal cord conduction velocity (SCCV) in rats can be determined in vivo by epidural stimulation of spinal cord with needle electrodes at the level of L1-L2 (proximal segment) and L5-L6 vertebrae (distal segment), and by recording the response of compound muscle action potentials (CMAPs) from the gastrocnemius muscle.

Using different methods to examine the particular aspects of any disorder would be beneficial for a better understanding of the pathology. Observation of the electrophysiological outcomes of SCI and calculation of conduction velocity can help in grading and quantifying the severity of SCI. According to our knowledge, epidural spinal cord stimulation-induced EMG activity has not been used in

${ }^{1}$ Department of Biophysics, Institute of Health Science, Adnan Menderes University Aydın, Aydın, Turkey; ${ }^{2}$ Department of Biophysics, Faculty of Medicine, Adnan Menderes University Aydın, Aydın, Turkey; ${ }^{3}$ Department of Cardiovascular Surgery, Faculty of Medicine, Adnan Menderes University Aydın, Aydın, Turkey; ${ }^{4}$ Department of Pathology, Faculty of Medicine, Adnan Menderes University Aydın, Aydın, Turkey and ${ }^{5}$ Department of Neurology, Faculty of Medicine, Adnan Menderes University Aydın, Aydın, Turkey Correspondence: H Basoglu, Department of Biophysics, Medical Faculty, Adnan Menderes University Aydın, Tip Fakültesi Dekanlik Binasi, Aydın 09100, Turkey. E-mail: hbasoglu@gmail.com

Received 19 October 2012; revised 22 March 2013; accepted 2 April 2013; published online 21 May 2013 
evaluation of SCI. The ischemia model induces a regional injury, which affects a wide range of spinal cord tissue. Therefore, we thought that an ischemia model would be more advantageous in determination of potential conduction velocity changes after SCI. In the present study, we aimed to observe the epidural spinal cord stimulationinduced EMG responses and investigate the possible alterations of SCCV and CMAPs after ischemic SCI in rats.

\section{MATERIALS AND METHODS}

The experimental designs and procedures were approved by the Animal Care Committee of Adnan Menderes University, and animals were treated in accordance with National Institute of Health's Guide for the Care and Use of Laboratory Animals. Male Sprague-Dawley rats (290-320 g) were fed a standard laboratory diet and water ad libitum, and housed at controlled room temperature $\left(24.5-25^{\circ} \mathrm{C}\right)$ and in a 12-h dark-light cycle before and during the study.

\section{Study groups and spinal cord ischemia model}

A total of 16 rats were randomly assigned to either of the control or spinal cord ischemia (ischemia group) groups, with eight animals in each group. Animals were anesthetized with intraperitoneal injection of ketamine hydrochloride $\left(100 \mathrm{mg} \mathrm{kg}^{-1}\right)$ and xylazine $\left(10 \mathrm{mg} \mathrm{kg}^{-1}\right)$, and were maintained on an additional dose of ketamine as required. Body temperature was monitored by using a rectal probe and was kept at $35.5-37.5^{\circ} \mathrm{C}$ with a heat lamp. Tail vein was cannulated for intravenous fluid replacement $(0.9 \% \mathrm{NaCl}$ solution), and tail artery was cannulated with a 24-gauge catheter for monitoring of distal arterial pressure and obtaining samples for blood gas analysis during the procedure. A single dose of intravenous cephazolin sodium $\left(10 \mathrm{mg} \mathrm{kg}^{-1}\right)$ was administered immediately before the surgery for infection prophylaxis. Spinal cord ischemia was induced by transient occlusion of the abdominal aorta according to the previously reported method. ${ }^{16}$ Briefly, animals were placed in prone position and a midline laparotomy was performed. The rats in the control group underwent laparotomy without aortic occlusion. In the ischemia group, the segment of aorta between the left renal artery and aortic bifurcation was exposed and intravenous heparin $\left(150 \mathrm{U} \mathrm{kg}^{-1}\right)$ was administered before aortic occlusion. The abdominal aorta was then occluded with vascular clamps (Vascu-Statt II-Scanlan, Saint Paul, MN, USA) for 45 min, placing one clamp just distal to the renal artery and the other proximal to the aortic bifurcation. The clamps and catheters were removed after $45 \mathrm{~min}$ and the abdomen was closed by using silk sutures in a double-layer manner. All animals were then returned to their cages for recovery with free access to food and water. Acetaminophen $\left(0.25 \mathrm{mg} \mathrm{ml}^{-1}\right)$ was added into the drinking water as a postoperative analgesic. The Crede maneuver was used to empty the urinary bladder twice a day.

\section{Neurological assessment}

The neurological assessment was performed $48 \mathrm{~h}$ after reperfusion by an independent observer when the animals were fully conscious. The motor functions of the rats were graded according to the modified Tarlov scoring system as follows: (0) no voluntary movement (complete paraplegia); (1) perceptible movement at the joint; (2) good joint mobility but unable to stand; (3) ability to stand but unable to walk; (4) weak walking; (5) complete recovery. ${ }^{17}$

\section{Electrophysiological studies}

Animals were re-anesthetized $48 \mathrm{~h}$ after surgery; a longitudinal midline dorsal incision was made and skin overlying the lumbosacral vertebrae was removed as a flap. Monopolar needle electrodes (EL400-Biopac System, Goleta, CA, USA) were used to stimulate spinal cord. Proximal stimulation electrodes were sequentially inserted at L1-L2 (L1 level) and L2-L3 (L2 level) dorsal intervertebral spaces. Distal stimulation electrodes were inserted in the same manner at L5-L6 (L5 level) and L6-S1 (L6 level) dorsal inter-vertebral spaces. Polarizations of stimulation electrodes were positive at L1 and L5 level and negative at L2 and L6 level. The skin surface of the left gastrocnemius muscle was shaved and cleaned with $70 \%$ ethanol. Four-mm-diameter shielded

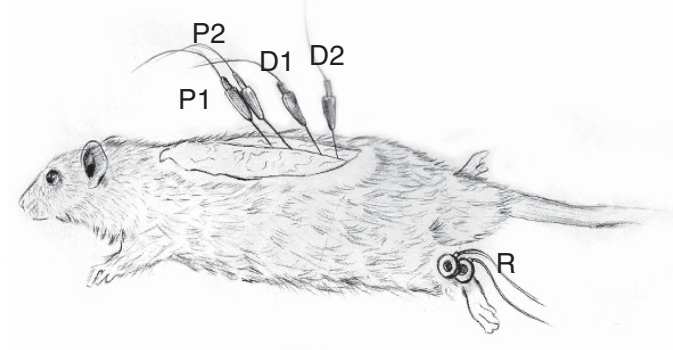

Figure 1 Positions of electrodes. P1: proximal stimulation electrode inserted at L1-L2 (L1 level) dorsal inter-vertebral space. P2: proximal stimulation electrode inserted at L2-L3 (L2 level) dorsal inter-vertebral space. D1: distal stimulation electrode at L5-L6 (L5 level) dorsal inter-vertebral space. D2: distal stimulation electrode at L6-S1 (L6 level) dorsal inter-vertebral space. R: surface recording electrodes on the surface of gastrocnemius muscle.

$\mathrm{Ag} / \mathrm{AgCl}$ surface recording electrodes (EL254S-Biopac System) were plastered to the skin surface of gastrocnemius muscle to obtain CMAPs (Figure 1). All stimulations and CMAPs records were processed by an MP100 data acquisition system (Biopac System).

The supramaximal stimulus is defined as a stimulus that has strength significantly higher than the required level to activate all the nerve fibers. In order to determine the supramaximal stimulus, sequential direct currents (DC) of $0.1,0.2,0.5,1,2,5,10,20$ and $50 \mathrm{~mA}$ were applied to epidural stimulation electrodes. A stimulus with a current of $\geqslant 5 \mathrm{~mA}$ yielded a unique and steady EMG trace; therefore, a supramaximal stimulus was defined as $\geqslant 5 \mathrm{~mA}$ in $10 \mathrm{~V}$ DC (Figure 2).

Analysis of spinal cord conduction velocity. A supramaximal electrical stimulus (intensity: $10 \mathrm{~V}$, current: $10 \mathrm{~mA} \mathrm{DC}$, duration: $0.1 \mathrm{~ms}$ ) was applied at first to proximal stimulation electrodes and then to distal stimulation electrodes. CMAPs were recorded from the gastrocnemius muscle for each stimulus (Figure 3). SCCV was calculated with the formula SCCV $=\Delta x / \Delta t$, where $\Delta x$ is the distance between the midpoint of proximal stimulation electrodes and the midpoint of distal stimulation electrodes, and $\Delta t$ is the latency difference of CMAPs that were recorded by distal and proximal stimulus. $\Delta x$ was measured by using a $120-\mathrm{mm}$ vernier caliper (CENCO company, Germany). Latency of CMAP is determined as the time interval between the stimulus artifact and the first deflection from the baseline of EMG signals (Figure 3).

Analysis of compound muscle action potentials. CMAP duration is defined as the time period from onset of the first negative deflection to return to the baseline of the last negative deflection (Figure 3). ${ }^{18}$ Amplitudes and durations of proximal and distal CMAPs were analyzed. Peak to peak ( $\mathrm{p}-\mathrm{p}$ ) values of CMAPs were used to analyze amplitudes. The time period between the onset and termination of action potentials was measured to identify the duration of CMAPs.

\section{Histopathologic evaluation}

Spinal cords of rats were harvested after SCCV measurement. Samples were fixed in $10 \%$ formalin and embedded in paraffin. Four serial transverse sections of $4 \mu \mathrm{m}$ thickness were obtained from L4-L6 spinal cord segments, stained with hematoxylin and eosin, selected randomly and examined under light microscopy $(\times 200)$. All samples were examined by a pathologist who was unaware of the experimental groups. The gray matter was evaluated for signs of ischemic injury. Neurons that displayed cellular swelling or eosinophilic cytoplasm, loss of nucleus and Nissl bodies were considered as injured. Cells that had a prominent nucleus with fine chromatin and cytoplasmic Nissl bodies were identified as viable cells. Viability index was calculated as the ratio between number of normal motor neurons and number of all motor neurons in the entire spinal cord section for each animal (viability index $=$ number of viable cells/total number of neurons). ${ }^{19}$ 


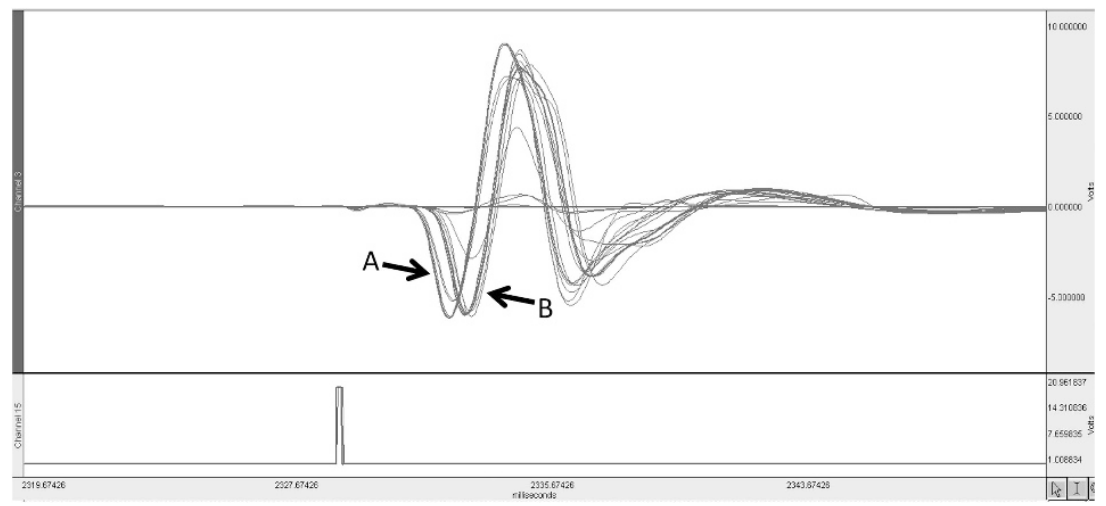

Figure 2 Overlapped images of EMG recordings (upper trace) obtained by proximal and distal epidural electrical stimulations (bottom trace) with direct currents of $0.1,0.2,0.5,1,2,5,10,20$ and $50 \mathrm{~mA}$. Bold lines indicate the superimposed EMG recordings for $5 \mathrm{~mA}$ and higher stimulations (Supramaximal stimuli); A: distal supramaximal recordings, B: proximal supramaximal recordings.

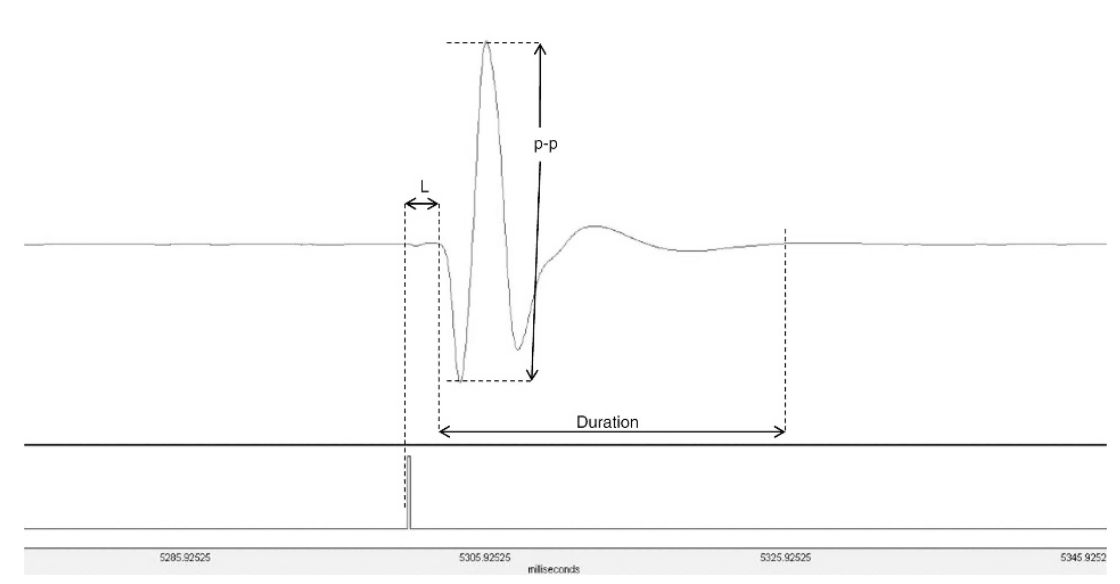

Figure 3 A typical EMG response of compound muscle action potential that was recorded immediately after supramaximal epidural stimulation of spinal cord. L: latency, Duration: duration of a CMAP, p-p: peak to peak value (amplitude).

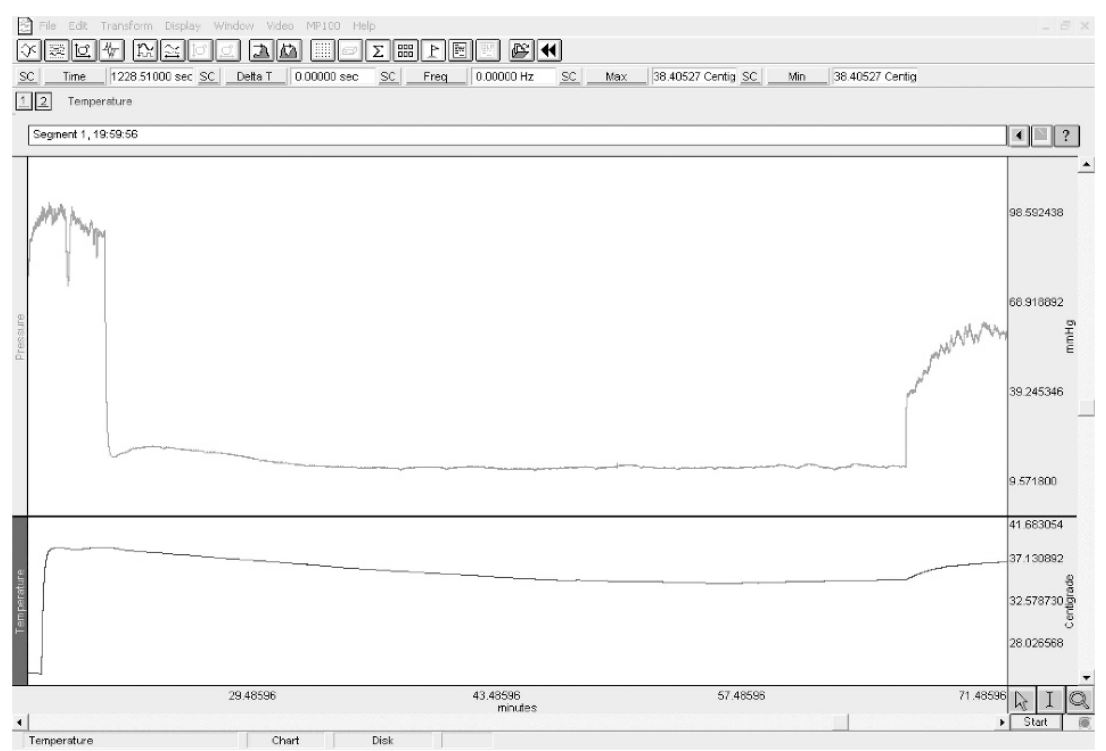

Figure 4 Representative recordings of rectal temperature (bottom trace) and arterial pressure (upper trace) during aortic occlusion. 


\section{Statistical analysis}

All data were presented as the mean \pm s.e.m. (standard error of mean), which was calculated using Graphpad Prism 5.0 (Graphpad, San Diego, CA, USA). The Mann-Whitney $U$-test was used to compare the neurologic assessment score and viability index among groups. Two-tailed unpaired $t$-test was used to compare spinal cord conduction velocities. $P<0.05$ level of probability was used as the criteria for significance.

\section{RESULTS}

There was no operative mortality in any of the groups and all animals survived throughout the study. The heart rate, arterial blood gases and $\mathrm{pH}$, blood pressure and body temperature were similar and stable in each group during the procedure. A rapid decrement in arterial blood pressure was observed immediately after clamping of the abdominal aorta in the ischemia group (Figure 4). The means of measured $\Delta x$ values (mean \pm s.d.) were $3.06 \pm 0.16$ and $3.01 \pm 0.16 \mathrm{~cm}$, respectively, for the control and the ischemia groups.

\section{Neurological outcome}

All rats in the control group had normal neurological functions. However, the rats in the ischemia group showed moderate to severe degrees of neurological deterioration following ischemia. Tarlov scores in the ischemia group were significantly reduced as compared with the control group (Table 1).

\section{Histopathological outcome}

Examination of the spinal cord samples in the control group showed normal structure of the gray matter. On the other hand, neurons in the ischemia group displayed variable degrees of neuronal injury (Figure 5). The viability indices of both the groups of rats are shown in Table 1.

Table 1 Tarlov scores, viability indices and spinal cord conduction velocity results in the control and the ischemia groups

\begin{tabular}{lcr}
\hline & Control group & Ischemia group \\
\hline Tarlov score & 5.0 & $1.5 \pm 0.4$ \\
Viability index (\%) & $91.9 \pm 1.3$ & $57.3 \pm 0.8$ \\
Spinal cord conduction velocity $\left(\mathrm{ms}^{-1}\right)$ & $103.1 \pm 2.7$ & $57.5 \pm 3.2$ \\
\hline
\end{tabular}

Data are presented as the mean \pm s.e.m. Values in the ischemia group are significantly different from the control group for Tarlov score, viability indices and spinal cord conduction velocity $(P<0.05)$.

\section{CMAPs and spinal cord conduction velocity}

The mean amplitudes of CMAPs in response to distal stimulations are $6.510 \pm 1.060 \mathrm{~V}$ for the control group and $4.499 \pm 0.559 \mathrm{~V}$ for the ischemia group. The amplitude in the ischemia group is smaller than the control group; however, the difference is statistically not significant. The mean durations of CMAPs generated by distal stimulations are $22.58 \pm 1.28$ and $77.70 \pm 52.55 \mathrm{~ms}$ for the control and ischemia groups, respectively; however, the difference is not significant because of high standard deviation.

The mean amplitudes of CMAPs evoked by proximal stimulations are $4.094 \pm 0.339 \mathrm{~V}$ for the control group and $4.788 \pm 0.540 \mathrm{~V}$ for the ischemia group. The mean durations of CMAPs induced by proximal stimulations are $23.97 \pm 1.45 \mathrm{~ms}$ for the control group and $25.26 \pm 1.69 \mathrm{~ms}$ for the ischemia group. The differences of both amplitudes and durations of CMAPs are not statistically significant in response to proximal stimulations between groups.

The means of calculated SCCV for both groups are shown in Table 1 . SCCV is found to be significantly decreased in the ischemia group as compared with the control group.

\section{DISCUSSION}

In the present study, spinal cord ischemia generated by aortic occlusion resulted in hind limb motor dysfunction, which was detected with significantly reduced Tarlov score in the ischemia group in comparison with the control group. Furthermore, histopathological examination revealed a significant reduction in the number of viable neurons in the ischemia group.

The mean amplitudes of CMAPs induced by distal stimulations in the ischemia group are slightly decreased as compared with the control group, and we also observed delayed potentials and dispersion in the ischemia group (Figure 6). Although statistically not significant, mean durations of CMAPs following distal stimulations in the ischemia group is more than 2 times longer than in the control group. The differences between the control and ischemia groups are too small regarding the amplitudes and durations of CMAPs in response to proximal stimulations, which suggested that these stimulations possibly travel directly through the sciatic nerve to produce an EMG response. We noticed a significant decrement in spinal cord conduction velocities determined by analysis of CMAPs in ischemic rats $(P<0.05)$, which is similar to the deterioration observed in neurobehavioral and histological evaluation.

Motor nerve conduction velocity is determined by stimulating a motor nerve at two distinct points and then analyzing CMAPs in peripheral nerves. ${ }^{20}$ This study investigates whether this basic approach can be adopted for analyzing spinal cord conduction in
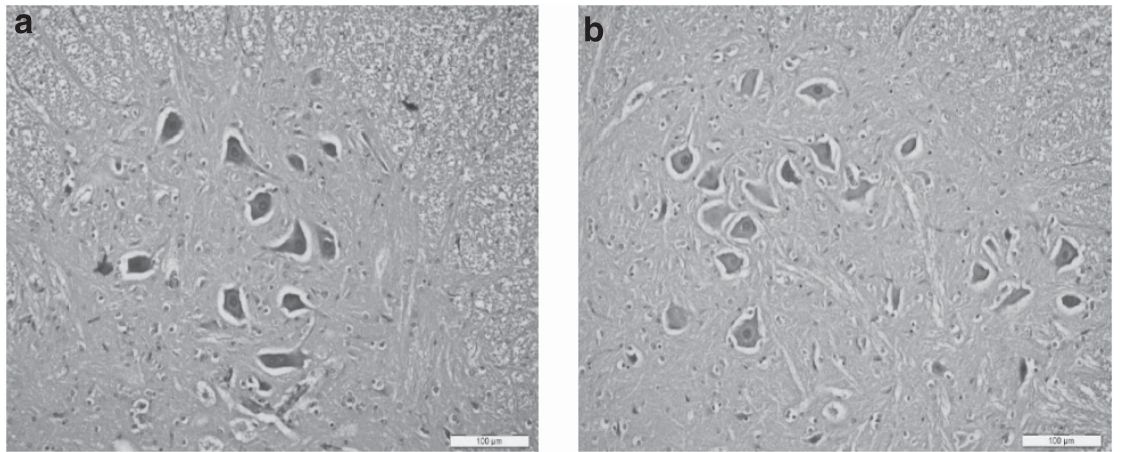

Figure 5 Representative histological samples from the control and the ischemia groups: The control group sample with no neuronal damage (a), the ischemia group neurons exhibiting cellular swelling, eosinophilic cytoplasm, loss of nucleus and Nissl bodies (b). 


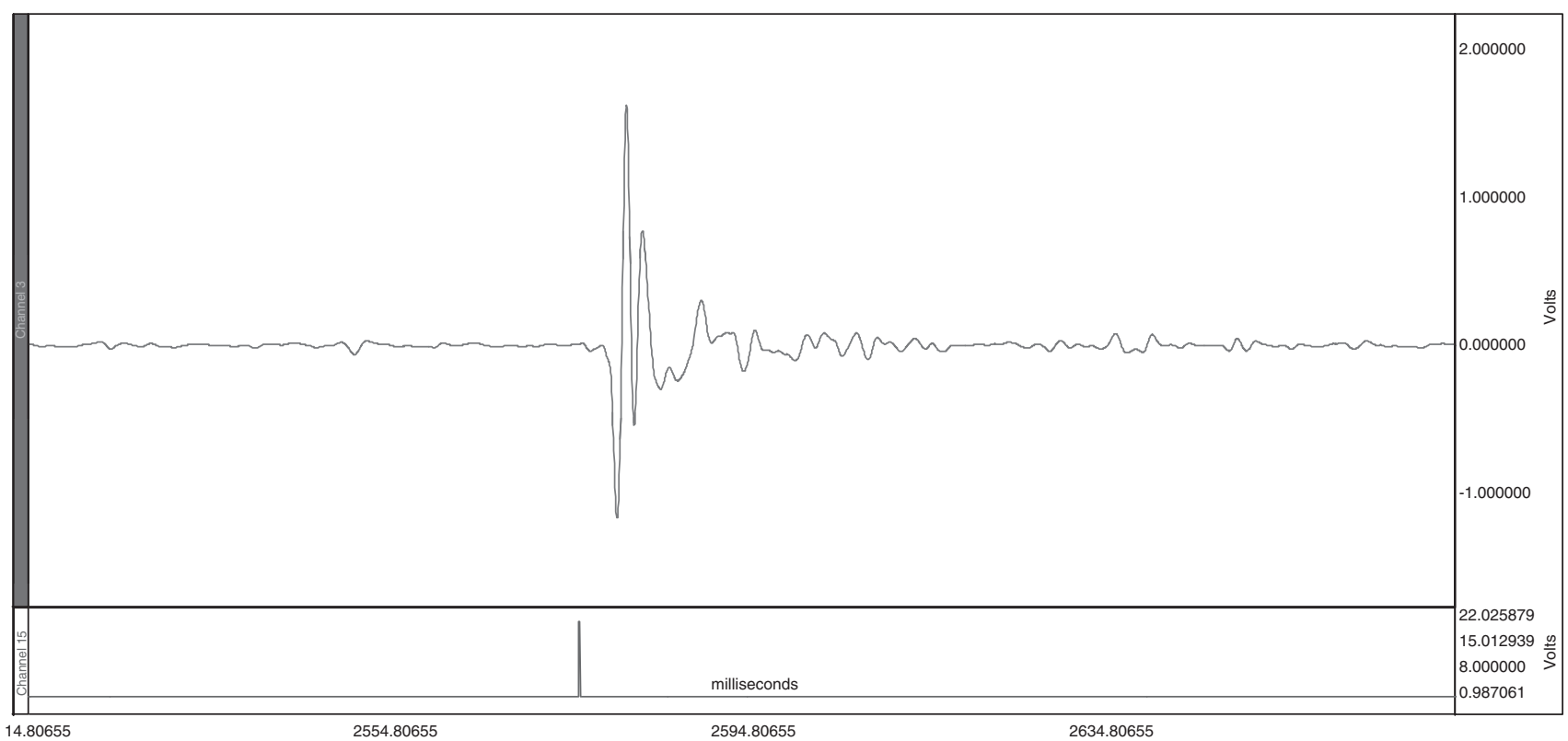

Figure 6 CMAP of an ischemic animal after distal epidural stimulation.

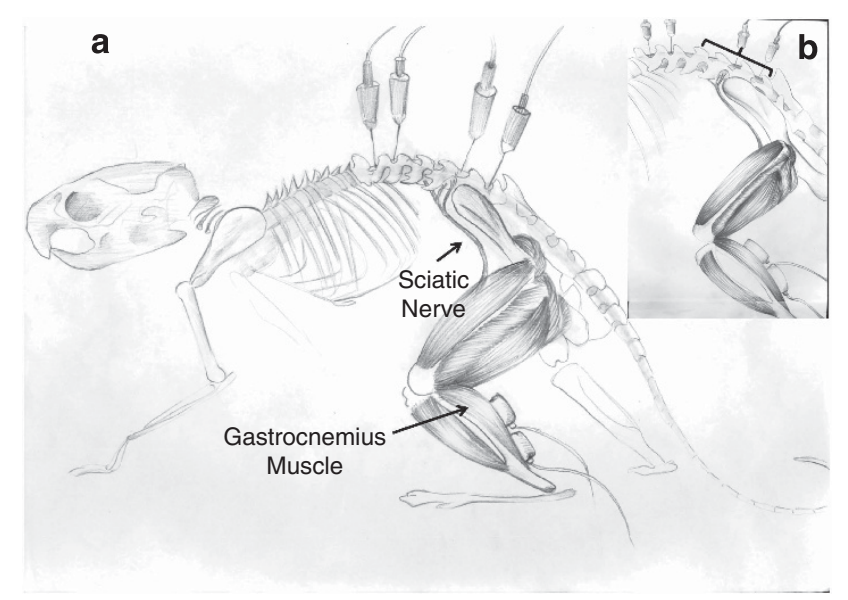

Figure 7 (a) Proximal and distal stimulation electrodes, sciatic nerve, gastrocnemius muscle and recording electrodes. (b) Bracket shows the experimental region of ischemia (L4-L6). Note that ischemic region is located between the proximal and distal stimulation electrodes.

spinal cord-injured rats. Supramaximal epidural stimulation fires all spinal cord neurons at the level of stimulation. We suggest that this stimulus generates action potentials that is transmitted through the spinal cord and pass to the sciatic nerve to produce CMAPs in the gastrocnemius muscle.

The sciatic nerve, which innervates the gastrocnemius muscle, is predominantly composed of nerve fibers originating from the spinal cord at the level of L4 and L5 vertebrae in rats $^{21}$ (Figure 7a). The ischemia produced via infrarenal aortic occlusion in rats induces injury at the L4-L6 segment of the spinal cord. ${ }^{16}$ Iliolumbar arteries originate from the abdominal aorta, caudal to the gonadal arteries in rats. Gonadal arteries are located caudal to the renal arteries; therefore the clamp was located below the renal but above the lumbar arteries. ${ }^{22}$ Therefore, the experimental region of injury is located between distal and proximal epidural stimulation points, and also covers the origin of the sciatic nerve (Figure $7 \mathrm{~b}$ ).
The L1-L2 stimulation could excite motor neurons through orthodromic activation of descending axons or antidromic activation of ascending pathways. L5-L6 stimulation could excite motor neuron axons directly, or activate nerve roots of the descending pathway and contract the gastrocnemius muscle. The EMG response (CMAPs) recorded following L1-L2 stimulation is believed to be the result of orthodromic activation that triggers gastrocnemius muscle contraction via the sciatic nerve. In spinal cord-transected rats at L4 level, CMAPs were abolished after L1-L2 stimulation, while CMAPs that are elicited by L5-L6 stimulation were still present (unpublished data; Figure 8). This observation is the evidence of activation of descending pathway via the L1-L2 stimulus. Therefore both the proximal and distal stimulation impulses travel through the experimental region of SCI and reach the sciatic nerve.

The alterations of SCCV following SCI detected in this study are thought to be primarily related with white matter damage. Both gray and white matter neurons of the spinal cord are susceptible to ischemic injury. Myelinated axons are essentially dependent on a continuous supply of oxygen and glucose. Ischemia-reperfusion can cause irreversible injury and result in serious central nervous system dysfunction, including the signal transmission..$^{23}$ It has been shown that both gray and white matter were damaged after spinal cord ischemia in rats, and the severity of white matter injury was correlated with the degree of gray matter injury. ${ }^{24,25}$ Kannellopoulos et al. ${ }^{26}$ demonstrated widespread and prominent vacuolation in white matter 2 days after the experimental spinal cord ischemia. The mechanism of injury induced by reoxygenation in axons has been proposed to be the failure of respiration due to severe mitochondrial $\mathrm{Ca}^{2}+$ overload. Although glia are relatively resistant to anoxia, oligodendrocytes and the myelin sheath may be damaged by glutamate released by reverse $\mathrm{Na}(+)$-glutamate transport. ${ }^{27}$ Although we did not specifically address the histological changes of white matter after ischemia in our study, we observed significant gray matter injury, which indicates that SCI was successfully induced by aortic occlusion.

The following limitations of the present study must be taken into account when considering the results. Exhibition of the 


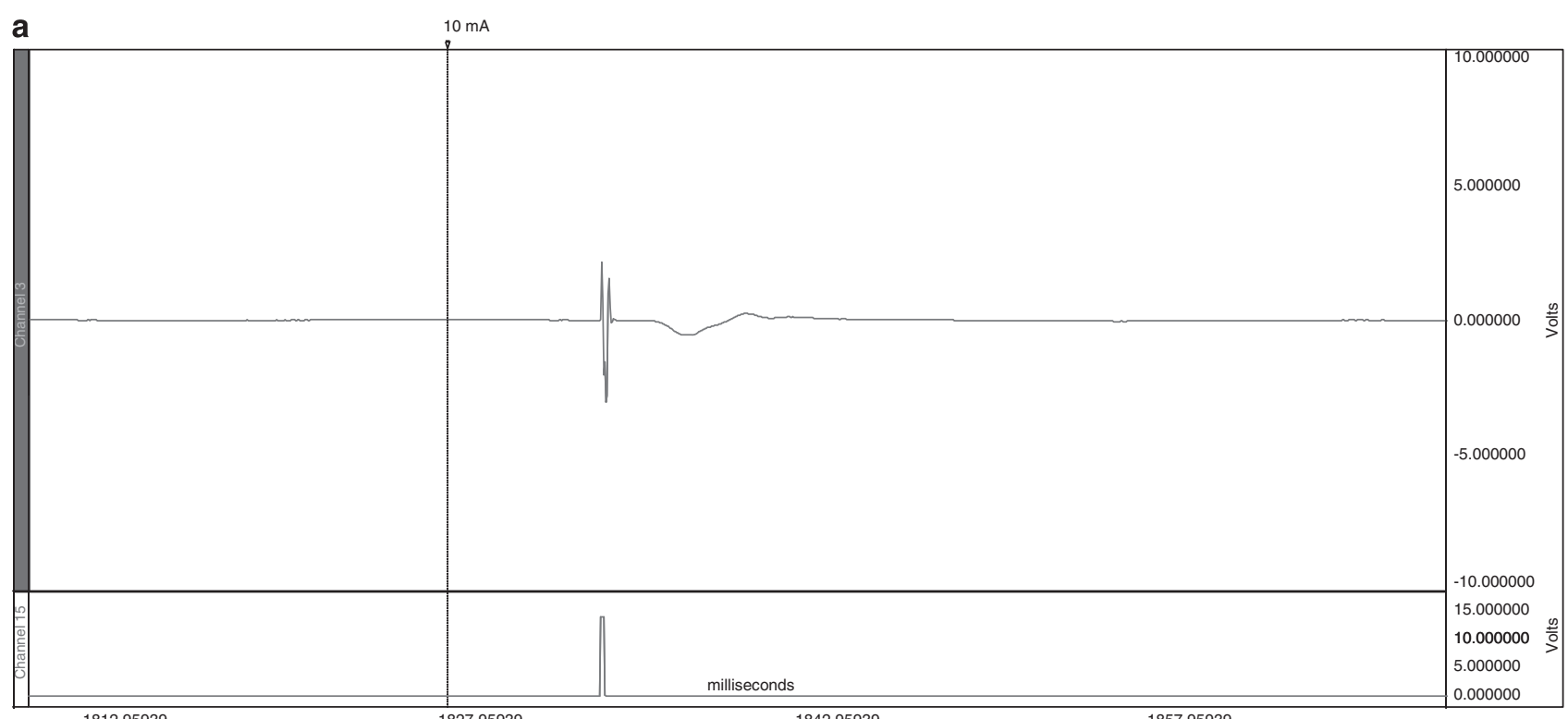
1827.95939 1842.95939

1857.95939

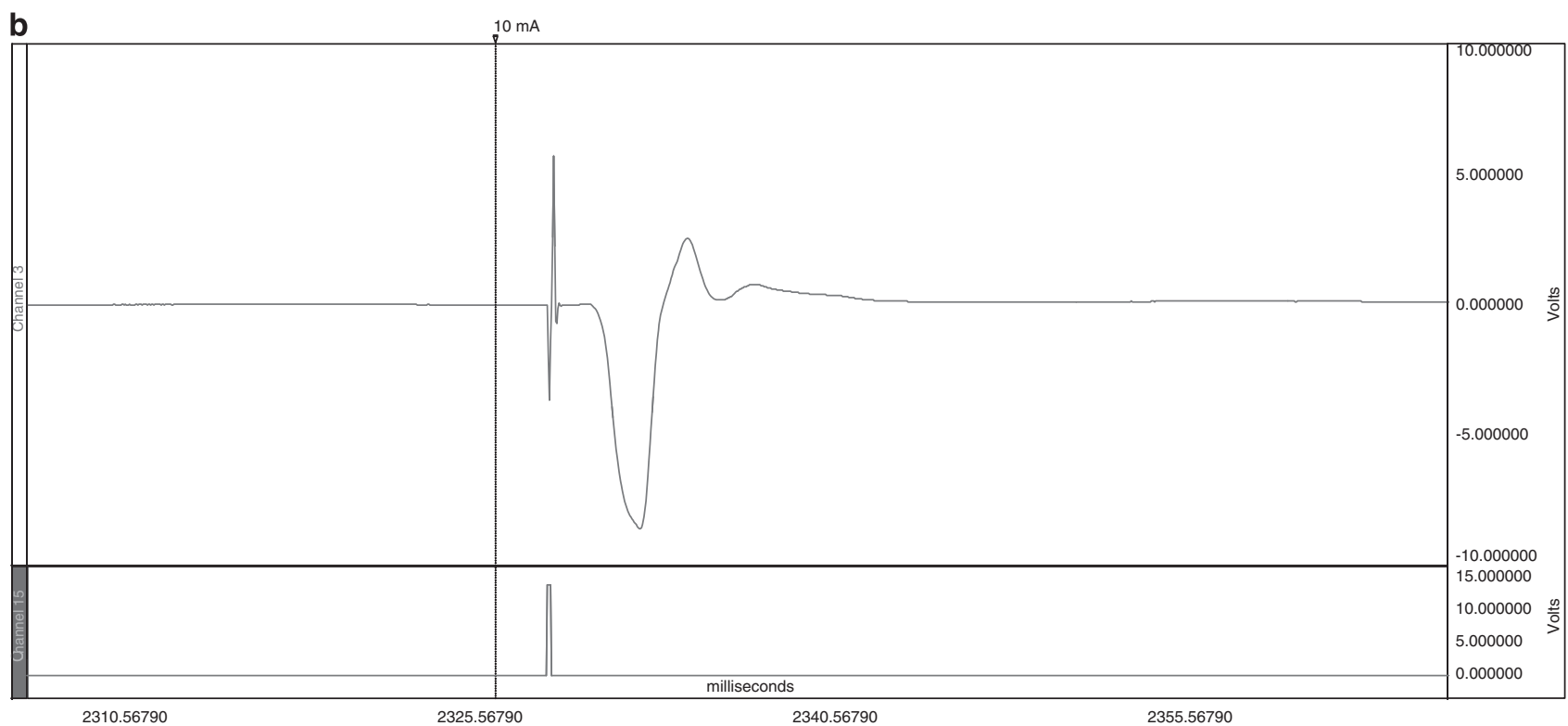

Figure 8 EMG recordings of a spinal cord transected rat at L4 level. (a) Proximal (L1-L2) supramaximal stimulation, (b) distal (L5-L6) supramaximal stimulation.

histopathological changes in white matter of the spinal cord could have provided a comprehensive understanding of the conduction alterations after spinal cord ischemia. Although the outcomes of locomotor scoring and SCCV show similar reduction in spinal cordinjured rats, multiple time-point measurements are needed to determine whether a correlation exists between these two parameters.

This study indicates that epidural spinal cord stimulation-induced EMG response can be obtained after SCI in rats. We observed a significant decline of SCCV $48 \mathrm{~h}$ after spinal cord ischemia, which is suggested to be related to white matter injury. The analysis of the CMAPs' amplitudes generated by distal stimulations showed partial reduction in the ischemia group compared with the control group. This finding may be the result of demyelination, ${ }^{18}$ axonal degeneration and/or loss of nerves in gray matter. In ischemic animals we also noticed dispersion of CMAPs and delayed potentials, which are supposed to be the result of myelin loss.
As a conclusion, measurement of SCCV via epidural electrical stimulation is possible and shows a significant decline after spinal cord ischemia in rats. We suggest that this method can aid in quantifying neurological damage after experimental ischemic SCI. However, it is necessary to investigate the alterations of SCCV in other experimental SCI models such as contusion, excitotoxic injury and so on.

\section{DATA ARCHIVING}

There were no data to deposit.

\section{CONFLICT OF INTEREST}

The authors declare no conflict of interest.

\section{ACKNOWLEDGEMENTS}

We would like to thank Umran Ayyildiz for illustrations. 
1 Nakae A, Nakai K, Yano K, Hosokawa K, Shibata M, Mashimo T. The animal model of spinal cord ınjury as an experimental pain model. J Biomed Biotechnol 2011; 2011 939023.

2 Kastner A, Gauthier P. Are rodents an appropriate pre-clinical model for treating spina cord injury? Examples from the respiratory system. Exp Neurol 2008; 213: 249-256.

3 Guertin PA. Semiquantitative assessment of hindlimb movement recovery without intervention in adult paraplegic mice. Spinal Cord 2005; 43: 162-166.

4 Hamers FP, Lankhorst AJ, van Laar TJ, Veldhuis WB, Gispen WH. Automated quantitative gait analysis during overground locomotion in the rat: its application to spinal cord contusion and transection injuries. J Neurotrauma 2001; 18: 187-201.

5 Semler J, Wellmann K, Wirth F, Stein G, Angelova S, Ashrafi M et al. Objective measures of motor dysfunction after compression spinal cord injury in adult rats: correlations with locomotor rating scores. J Neurotrauma 2011; 28: 1247-1258.

6 Nessler JA, De Leon RD, Sharp K, Kwak E, Minakata K, Reinkensmeyer DJ. Robotic gait analysis of bipedal treadmill stepping by spinal contused rats: characterization of intrinsic recovery and comparison with BBB. J Neurotrauma 2006; 23: 882-896.

7 Robertson G, Caldwell G, Hamill J, Kamen G, Whittlesey S. Electromyographic kinesiology. In: Robertson LD (ed) Research Methods in Biomechanics. Human Kinetics Publ.: Champaign, IL, USA, 2004, pp 163-181.

8 Ballermann M, Tse AD, Misiaszek JE, Fouad K. Adaptations in the walking pattern of spinal cord injured rats. J Neurotrauma 2006; 23: 897-907.

9 Majczyński H, Maleszak K, Górska T, Sławińska U. Comparison of two methods for quantitative assessment of unrestrained locomotion in the rat. J Neurosci Methods 2007; 163: 197-207.

10 Gerasimenko Y, Roy RR, Edgerton VR. Epidural stimulation: comparison of the spinal circuits that generate and control locomotion in rats, cats and humans. Exp Neurol 2008; 209: 417-425.

11 Dougherty JB, Goodman JM, Knudsen EB, Moxon KA. Controlled unilateral isometric force generated by epidural spinal cord stimulation in the rat hindlimb. IEEE Trans Neural Syst Rehabil Eng 2012; 20: 549-556.

12 Park JP, Kim KJ, Phi JH, Park CK, Kim JH, Kang HJ et al. Simple measurement of spinal cord evoked potential: a valuable data source in the rat spinal cord injury model. J Clin Neurosci 2007; 14: 1099-1105.

13 Barchini J, Tchachaghian S, Shamaa F, Jabbur SJ, Meyerson BA, Song Z et al. Spinal segmental and supraspinal mechanisms underlying the pain-relieving effects of spinal cord stimulation: an experimental study in a rat model of neuropathy. Neuroscience 2012; 215: 196-208.
14 Magnuson DSK, Trinder TC, Zhang YP, Burke D, Morassutti DJ, Shields CB. Comparing deficits following excitotoxic and contusion injuries in the thoracic and lumbar spinal cord of the adult rat. Exp Neurol 1999; 156: 191-204.

15 Kose $\mathrm{H}$, Basoglu $\mathrm{H}$. Analysis of spinal nerve conduction measurement with epidural stimulation in rats. J Neurol Sci (Turkish) 2010; 27-2: 112

16 Isbir CS, Ak K, Kurtkaya O, Zeybek U, Akgün S, Scheitauer BW et al. Ischemic preconditioning and nicotinamide in spinal cord protection in an experimental model of transient aortic occlusion. Eur J Cardiothorac Surg 2003; 23: 1028-1033.

17 Gong G, Yuan LB, Hu L, Wu W, Yin L, Hou JL et al. Glycyrrhizin attenuates rat ischemic spinal cord injury by suppressing inflammatory cytokines and HMGB1. Acta Pharmacol Sin 2012; 33: 11-18.

18 Tankisi H, Otto M, Pugdahl K, Johnsen B, Fuglsang-Frederiksen A. Correlation between compound muscle action potential amplitude and duration in axonal and demyelinating polyneuropathy. Clin Neurophysiol 2012; 123: 2099-2105.

19 Boga M, Discigil B, Ozkisacik EA, Gurcun U, Badak MI, Dikicioglu E et al. The combined effect of iloprost and $\mathrm{N}$-acetylcysteine in preventing spinal cord ischemia in rabbits. Eur J Vasc Endovasc Surg 2006; 31: 366-372.

20 Kane NM, Oware A. Nerve conduction and electromyography studies. J Neurol 2012 259: 1502-1508.

21 Rigaud M, Gemes G, Barabas ME, Chernoff DI, Abram SE, Stucky CL et al. Species and strain differences in rodent sciatic nerve anatomy: Implications for studies of neuropathic pain. Pain 2008; 136: 188-201.

22 Wingerd BD. Rat Dissection Manual. John Hopkins University Press: Baltimore, MD, USA, 1988, , p 59.

23 Stys PK. White matter injury mechanisms. Curr Mol Med. 2004; 2: 113-130.

24 Kurita N, Kawaguchi M, Horiuchi T, Inoue S, Sakamoto T, Nakamura M et al. An evaluation of white matter injury after spinal cord ischemia in rats: a comparison with gray matter injury. Anesth Analg 2005; 100: 847-854.

25 Takeda M, Kawaguchi M, Kumatoriya T, Horiuchi T, Watanabe K, Inoue S et al. Effects of minocycline on hindlimb motor function and gray and white matter injury after spinal cord ischemia in rats. Spine 2011; 36: 1919-1924.

26 Kanellopoulos GK, Xu XM, Hsu CY, Lu X, Sundt TM, Kouchoukos NT. White matter injury in spinal cord ischemia: protection by AMPA/kainate glutamate receptor antagonism. Stroke 2000; 31: 1945-1952.

27 Stys PK. Anoxic and ischemic injury of myelinated axons in CNS white matter: from mechanistic concepts of therapeutics. J Cereb Blood Flow Metab 1998; 18 $2-25$. 